\title{
Breath Holding Predicts Reduced Smoking Intake but Not Quitting
}

\author{
David Welch ${ }^{*}, 1$ and Rob McGee ${ }^{2}$ \\ ${ }^{1}$ School of Population Health, University of Auckland, Auckland, New Zealand \\ ${ }^{2}$ Department of Preventive and Social Medicine, Dunedin School of Medicine, University of Otago, Dunedin, New \\ Zealand
}

\begin{abstract}
Introduction: Cigarette smokers who attempt to quit may or may not relapse. Previous research has shown that breath-holding ability predicts successful quitting, and it has been suggested that breath-holding ability may be a marker of tolerance for uncomfortable physiological symptoms.
\end{abstract}

Methods: We measured breath-holding ability and smoking in a large general-population birth cohort from age 21 to age 32.

Results: Cross-sectionally, at age 21, breath-holding ability was greater in males who had quit smoking than in either current or non-smokers, while no such differences were observed amongst females. On the other hand, no prospective associations were detected between breath-holding ability and quitting smoking by age 32. Pack years smoked was lower amongst male smokers with better breath-holding ability at age 32 .

Discussion: While the cross-sectional finding in males was consistent with the hypothesis that those with more tolerance for breath holding would be more successful at quitting, there was no long-term effect to age 32 . On the other hand, breath-holding was associated with reduced intake over time in males. A behavioural mechanism whereby longer breath holders smoke less because of a greater tolerance for the physical discomfort associated with intervals between cigarettes is consistent with these findings.

Keywords: Relapse prevention, epidemiology, longitudinal research, smoking.

\section{INTRODUCTION}

Cigarette smokers who attempt to quit often relapse. It has been hypothesised that the desire to smoke again comes from anticipated negative reinforcement associated with the reduction in withdrawal symptoms [1]. Once a person yields to the desire, the anticipated negative reinforcement becomes real, as craving is reduced, and relapse occurs.

The mechanisms underlying why some people manage to withstand the desire to smoke again and others not are complex, and have been reviewed elsewhere [2]; we have focussed on one aspect of this. Individual differences in breath-holding ability have been considered as a proxy for capacity to tolerate the negative affect associated with withdrawal symptoms [3]. This study found that people who could hold their breath for longer had a greater rate of success at stopping smoking after a course of antismoking treatment. In another study, it was determined that breathholding ability correlated with grip-holding ability [4], a finding which gave validity to the measure and implied a more general trait of endurance for physical discomfort, of which both measures were markers.

Length of breath holding has subsequently been used as a measure of tolerance of negative affect from physiological

\footnotetext{
*Address correspondence to this author at the School of Population Health, Faculty of Medical and Health Sciences, University of Auckland, Private Bag 92019, Auckland 1142, New Zealand; Tel: + 649 9238404; Fax: +64 9
} 3737496; Email: d.welch@auckland.ac.nz symptoms of smoking withdrawal. For example, breath holding ability was compared between groups of smokers who had previously abstained for long (seven days or more) or short (less than seven days); it did not differ [5]. More extreme groupings of smokers: smokers who had previously relapsed within a day at best $v$ s those who had previously sustained quitting for three months or longer prior to relapse, on the other hand, did show a difference in breath-holding ability whereby sustained quitters held their breaths for longer [6].

Previous research has considered the cross-sectional association between breath holding and smoking abstinence in small convenience samples. We considered the crosssectional relationship in a larger general population birth cohort at age 21 to test the hypothesis that longer breath holding would be associated with a greater rate of quitting smoking. Previous research has not established whether breath-holding ability predicts long-term abstinence, but it is reasonable to suppose that a greater ability to avoid relapse in the short term would lead to a reduced rate of smoking in the long term. We examined the association between breath holding at age 21 and smoking at age 32 to test the hypothesis that longer breath holding at age 21 would predict reduced likelihood of smoking 11 years later. Thirdly, greater tolerance for physical discomfort may allow smokers to wait longer between cigarettes, and thus to have lower intake over time. We examined the correlation between breath-holding ability and lifetime pack years smoked at ages 21 and 32 to test this hypothesis both cross-sectionally and longitudinally. 


\section{MATERIALS AND METHODOLOGY}

\section{Participants}

Participants were members of the Dunedin Multidisciplinary Health and Development Study, a longitudinal investigation of health and behaviour in a cohort born in Dunedin, New Zealand between April 1972 and March 1973 [7]. The base sample for the study was 1037 children ( $91 \%$ of eligible births; $52 \%$ male) who participated in the first follow-up assessment at age 3. Their families represented the full range of socioeconomic status in the general population of New Zealand's South Island and were mainly of European descent. Further follow-up assessments were done at ages $5,7,9,11,13,15,18,21,26$ and most recently at age 32 years, when we assessed $972(96 \%)$ of the 1015 living study members. The Otago Ethics Committee granted approval for each phase of this study and participants gave informed consent.

\section{Breath Holding}

Breath-holding ability at age 21 was assessed by timing study members while they held their breath for as long as possible, as described in previous research [3]. This assessment formed part of a broader assessment of smoking behaviours at this age.

\section{Smoking Status}

Smoking status was assessed by self-report in an interview at ages 21 and 32, and was coded as "current smoker", "ex-smoker", or "non-smoker" at each age. To be classified as smokers or ex-smokers, participants had to have smoked 20 packs of cigarettes, or at least one cigarette per day for as long as a year during their lives [8]. Current smokers were those who reported currently smoking at interview. Because we wanted to compare our results with data from cross-sectional studies, at age 21, ex-smokers were defined as those who reported being non-smokers at interview, but who had previously been smokers. At age 32, on the other hand, we required smokers to have quit at least a year prior to the day of assessment in order to categorise them as ex-smokers. The group who fell in this grey area of not smoking at interview but having smoked within the last year $(\mathrm{N}=40)$ were excluded from the analysis on smoking status.

\section{Pack Years Smoked}

Information about daily consumption of cigarettes and duration of that level of consumption was collected at ages $18,21,26$, and 32, with lifetime exposure assessed at age 18 . This information was used to estimate the number of pack years ( $=20$ cigarettes per day for a year) smoked by each study member up to age 21 , and up to age 32 .

\section{Analyses}

Preliminary analyses showed that breath-holding data were reasonably normally distributed. Two sets of analyses were carried out, the cross-sectional and the prospective, and for each, two approaches were taken. The first approaches compared the mean breath-holding ability of those who had quit smoking with other groups, to test the hypothesis that smokers who had quit smoking would have longer breathholding ability than smokers who continued to smoke. The second approach tested for associations between pack years smoked and breath-holding ability; negative associations would be consistent with the hypothesis that people who held their breath for longer would tend to have reduced intake of cigarettes over time.

For the cross-sectional analysis at age 21, mean breathholding ability was compared between current, ex-, and nonsmoker groups using one-way analysis of variance. Secondly, the correlation coefficient between pack years smoked and breath-holding ability was calculated for all those who had smoked by age 21 .

For the prospective analysis to age 32 , two-way analysis of variance was used, treating smoking at ages 21 and 32 as two factors, and breath holding as the dependent variable. In the second stage of this analysis, the correlation coefficient was calculated for the association between pack years smoked to age 32 and earlier breath-holding ability for all those who had smoked by age 32 .

Table 1. Change and Continuity in Smoking Status in the Cohort at Ages 21 and 32

\begin{tabular}{|c|c|c|c|c|c|}
\hline \multicolumn{2}{|c|}{} & \multicolumn{4}{|c|}{ Age 21 } \\
\cline { 3 - 6 } \multicolumn{2}{|c|}{} & Non-Smoker & Ex-Smoker & Smoker & Total \\
\hline \hline \multirow{4}{*}{ Age 32 } & Non-smoker & $\begin{array}{c}462 \\
(90.4 \%)\end{array}$ & 0 & 0 & $\begin{array}{c}462 \\
(49.2 \%)\end{array}$ \\
\cline { 2 - 6 } & Ex-smoker & $\begin{array}{c}14 \\
(2.7 \%)\end{array}$ & $\begin{array}{c}66 \\
(66.7 \%)\end{array}$ & $\begin{array}{c}68 \\
(20.7 \%)\end{array}$ & $\begin{array}{c}148 \\
(15.8 \%)\end{array}$ \\
\cline { 2 - 6 } & Smoker & $\begin{array}{c}35 \\
(6.8 \%)\end{array}$ & $\begin{array}{c}33 \\
(33.3 \%)\end{array}$ & $\begin{array}{c}261 \\
(79.3 \%)\end{array}$ & $\begin{array}{c}329 \\
(35.0 \%)\end{array}$ \\
\cline { 2 - 6 } & Total & 511 & 99 & 329 & 939 \\
\hline
\end{tabular}

\section{RESULTS}

\section{Smoking Behaviour at 21 and 32}

Data were available at both ages from 939 participants (Table 1). At age 21, 54.4\% were classified as non-smokers, $10.5 \%$ were ex-smokers, and $35.0 \%$ were current smokers. Of the smoking groups at 21 , most non-smokers $(90.4 \%)$ had never smoked by age 32 , while most smokers $(79.3 \%)$ still smoked. Of the ex-smokers at $21,33.3 \%$ were smoking at age 32 (Table $\mathbf{1}$ ).

There was no difference in the smoking status of males and females at age 21 (Chi-squared ( 2 d.f.) $=3.13, p=0.208$ ), but more males smoked at age 32 and more females were exsmokers (Chi-squared $(2$ d.f. $)=12.32, \quad \mathrm{p}=0.002)$. Male smokers (mean=3.8, $\mathrm{SD}=3.1$ ) were marginally higher than females (mean $=3.3, \mathrm{SD}=2.5$ ) in pack years smoked at age 21 $(\mathrm{t}(448)=1.81, \mathrm{p}=0.072)$. Males' pack years $(\mathrm{mean}=10.5$, $\mathrm{SD}=6.7)$ were also higher than females' (mean=8.4, $\mathrm{SD}=5.9)$ at age $32(\mathrm{t}(492)=3.77, \mathrm{p}<0.001)$. At age 21 , males $($ mean $=$ $61.0 \mathrm{~s}, \mathrm{SD}=20.0)$ held their breath for longer than females (mean $=40.4 \mathrm{~s}, \mathrm{SD}=14.2)$, with $\mathrm{t}(924)=18.01, \mathrm{p}<0.001$.

\section{Cross-Sectional Analysis at Age 21}

Since there were differences between the sexes in terms of both breath holding and smoking, sex was included as a 
factor in the analysis of variance model used. An initial model detected a marginal interaction between smoking status and $\operatorname{sex}(\mathrm{F}(2,901)=2.363, \mathrm{p}=0.095)$, so further analyses were stratified on the basis of sex.

Amongst males, there was a difference between the smoking status groups $(\mathrm{F}(2,458)=3.582, \mathrm{p}=0.029)$ in that exsmokers could hold their breaths for longer (mean $=68.7 \mathrm{~s}$, $\mathrm{SD}=20.3$ ) than smokers (mean $=60.8 \mathrm{~s}, \mathrm{SD}=19.4)$ or nonsmokers (mean=59.9s, SD=20.0). No differences between smoker status groups were detected for females $(\mathrm{F}(2,443)=0.036, \mathrm{p}=0.965)$.

The pack years data were non-normally distributed, and this led to skewness in the residuals of the linear function, so the Spearman's Rho coefficient was calculated for the relationship between pack years and breath holding. Given the observed sex differences in smoking status and breath holding, this was calculated separately for males (Rho=$0.114, \mathrm{p}=0.109)$ and females $(\mathrm{Rho}=-0.082, \mathrm{p}=0.227)$.

\section{Prospective Analysis to Age 32}

As in the cross-sectional analysis, sex was factored into the model. There were no differences in smoking status group means, or interaction effects detected by the model. In particular, there was no main effect of smoking status at age $32(\mathrm{~F}(1,899)=0.164, \mathrm{p}=0.685)$, or interaction between smoking status at 21 and $32(\mathrm{~F}(1,899=0.501, \mathrm{p}=0.479)$. The lack of these effects suggests that breath-holding ability was not different between smokers, smokers who had quit, or non-smokers (Fig. 1).

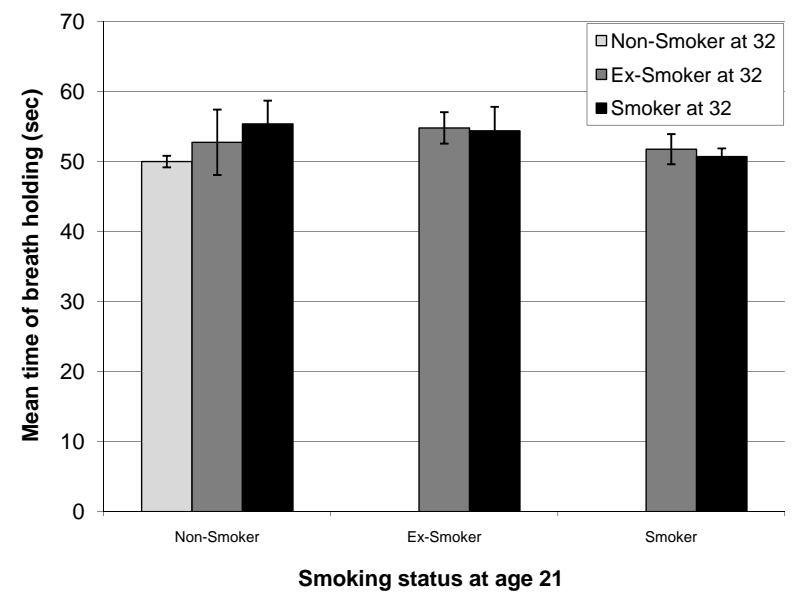

Fig. (1). Mean breath-holding ability of non-smokers, ex-smokers, and current smokers at age 21 divided according to their smoking status at age 32. Values graphed are marginal means including statistical control for sex. Error bars represent one standard error of the mean.

The residuals for the linear relation between lifetime pack years to age 32 and breath-holding ability were less severely skewed than at age 21 , however the non-parametric association is presented for comparability. Once again, and for the same reasons, analyses were conducted separately by sex, and were restricted to those who had smoked at some stage. Males $(\mathrm{Rho}=-0.156, \mathrm{p}=0.020)$ but not females $(\mathrm{Rho}=-$ $0.036, p=0.578)$ showed an association wherein those male smokers who could hold their breath for longer tended to have lower pack years smoked

\section{DISCUSSION}

We tested whether breath holding, as a proxy measure of tolerance to physiological discomfort, predicted quitting smoking. We found that 1) it was associated with quitting smoking in men in the short term, but 2) it did not predict quitting smoking in the long term. On the other hand, 3) it did predict a lower smoking intake amongst men who smoked.

\section{Cross-Sectional Analyses}

At age 21, male ex-smokers were able to hold their breath for slightly longer than other males, though no effects were seen for females. Possibly, those males who had managed to quit prior to age 21 were more tolerant of physiological distress and had thus been able to succeed in quitting, and/or the process of quitting may have fostered the development of this tolerance. Why the effect should have appeared in males but not females is unclear.

We also considered the correlation between pack years smoked and breath holding amongst those who had ever smoked by age 21 . There were small negative correlations which did not have the magnitude to be detected at conventional probability levels, but the direction of these was to suggest that smokers who could hold their breath for longer tended to have lower smoke intake.

\section{Prospective Analyses}

It was hypothesised that smokers who had successfully quit smoking by age 32 would have tended to possess longer breath-holding ability because they would have been less likely to relapse after quitting. If this had been so, the mean breath-holding ability for the group who no longer smoked would have been higher than for the group who still smoked. The lack of a detectable interaction between smoking at 21 and 32 in terms of breath-holding ability implies that this is not true. Secondary analyses were conducted in which smokers at 21 were limited to those who smoked moderately or more $(13+$ cigarettes per day), or heavily $(18+$ per day) in case the hypothesised effects were only observable in heavier smokers. This was not the case, and no difference in breath-holding ability was observed in those who had quit compared with those who continued to smoke.

On the other hand, males (but not females) showed a negative correlation between their lifetime pack years smoked and breath holding. This implies that there may be an effect of breath holding (or its underlying causal factor) on smoking. The findings are consistent with the idea that men who could tolerate physical discomfort for longer smoked less because they could tolerate discomfort of shortterm 'abstinences' between cigarettes. If this is so, it is unclear why it was not also true of women; one possibility is that women who had children may have altered their smoking patterns in a manner inconsistent with their breath holding, thus obscuring the effect. To test this, a secondary analysis was conducted, restricted to just those women $(\mathrm{N}=107)$ who had smoked, but who had not had children by age 32 , but no effect was detected $(\mathrm{Rho}=0.067, \mathrm{p}=0.493)$.

What exactly breath-holding ability measures is unknown. It is a psychophysiological phenomenon and is thus influenced by many factors, though the notion that it 
might partly reflect capacity to endure physical discomfort $[3,4]$ is of interest to smoking researchers. Our findings suggest that, if it does reflect this, the capacity is not related to completely quitting smoking in the long term, but that it may reflect a reduction in the short-term tendency to desire or require a cigarette.

\section{Strengths and Limitations}

This is the first study to investigate breath holding and smoking behaviour in a large general-population birth cohort. A major strength of this research is the generalisability of the findings to smokers throughout the western world. Furthermore, the long-term prospective nature of the study adds a dimension to the research that previous cross-sectional studies could not; it allows us to consider the effects across a period of 11 years, a significant proportion of a lifetime.

One limitation of the study is that we did not record intent to quit, so cannot comment directly on the relevance of breath holding to continued abstinence. On the other hand, there is no reason to suppose that intent to quit would be related to breath holding, so the effects of this should even out across the group. Nonetheless, inclusion of intent to quit smoking would add to the picture painted here.

A second limitation is the lack of detail in terms of when and how each individual smoked, abstained from smoking, and relapsed to smoking. This microstructure of the smokers' behaviour is not easily collected by large-scale epidemiological studies, but would be valuable for interpreting the findings described here. Our pack year findings were consistent with the idea that male smokers with greater endurance of physical discomfort remained in abstinence for longer between cigarettes, but we cannot know the detailed reflection of this in a smoker's daily life. For example, do they smoke at a slower regular rate, or have long-term abstinences, then smoke at a normal rate when they do smoke. This limitation could be addressed by more in-depth study of a smaller group of smokers.

\section{CONCLUSION}

Cross-sectionally, at age 21, male ex-smokers were able to hold their breath for longer than other males, a finding consistent with the hypothesis that breath-holding ability is a proxy for tolerance of physiological discomfort. On the other hand, age- 21 breath-holding ability was not predictive of having quit smoking by age 32 . In terms of the total amount smoked, however, there were marginal effects at age 21 , which were stronger by age 32, showing lower pack years in men with greater breath-holding ability. This finding is consistent with a mechanism where breath holding is a proxy for endurance of physical discomfort, and this endurance promotes abstinence in the face of uncomfortable withdrawal symptoms. How the effect observed is mirrored in reduced daily intake is unknown and would be an interesting topic for future research. From a clinical perspective, our findings suggest that ability to quit smoking in the long term may have more to do with other factors such as interpersonal support, and not 'physical toughness' or ability to go it alone.

\section{ACKNOWLEDGEMENTS}

The Dunedin Multidisciplinary Health and Development Research Unit is supported by the Health Research Council of New Zealand. We thank the Dunedin Study members and their parents, Unit research staff, Richie Poulton the current director, and the Study founder, Phil Silva.

\section{REFERENCES}

[1] Baker TB, Piper ME, McCarthy DE, et al. Addiction Motivation Reformulated: an affective processing model of negative reinforcement. Psychol Rev 2004; 111(1): 33-51.

[2] Brown RA, Lejuez CW, Kahler CW, et al. Distress tolerance and early smoking lapse. Clin Psychol Rev 2005; 25(6): 713-33.

[3] Hajek P, Belcher M, Stapleton J. Breath-holding endurance as a predictor of success in smoking cessation. Addict Behav 1987; 12: 285-8.

[4] Hajek P. Breath holding and success in stopping smoking: what does breath holding measure? Int J Addict 1989; 24(7): 633-9.

[5] Zvolensky MJ, Feldner MT, Eifert GH, et al. Affective style among smokers - Understanding anxiety, sensitivity, emotional reactivity, and distress tolerance using biological challenge. Addict Behav 2001; 26(6): 901-15.

[6] Brown RA, Lejuez CW, Kahler CW, et al. Distress tolerance and duration of past smoking cessation attempts. J Abnorm Psychol 2002; 111(1): 180-5.

[7] Silva PA, Stanton WR, Eds. From child to adult: the dunedin multidisciplinary health and development study. Auckland, NZ: Oxford University Press 1996.

[8] Ferris BG. Epidemiology standardization project. Am Rev Respir Dis 1978; 118: 1-120. 